\title{
New families of alternating harmonic number sums
}

\author{
Anthony Sofo
}

Victoria University, P. O. Box 14428, Melbourne City, Victoria 8001, Australia

E-mail: anthony.sofo@vu.edu.au

\section{Abstract}

We develop new closed form representations of sums of alternating harmonic numbers and reciprocal binomial coefficients.

2010 Mathematics Subject Classification. 05A10. 05A19, 33C20

Keywords. Combinatorial series identities, Summation formulas, Partial fraction approach, Alternating harmonic numbers, Binomial coefficients, Integral representation.

\section{Introduction and Preliminaries}

Let $\mathbb{R}$ and $\mathbb{C}$ denote respectively, the sets of real and complex numbers and let $\mathbb{N}:=\{1,2,3, \cdots\}$ be the set of positive integers, and $\mathbb{N}_{0}:=\mathbb{N} \cup\{0\}$. A generalized binomial coefficient $\left(\begin{array}{l}\lambda \\ \mu\end{array}\right)(\lambda, \mu \in \mathbb{C})$ is defined, in terms of the familiar (Euler's) gamma function, by

$$
\left(\begin{array}{l}
\lambda \\
\mu
\end{array}\right):=\frac{\Gamma(\lambda+1)}{\Gamma(\mu+1) \Gamma(\lambda-\mu+1)}, \quad(\lambda, \mu \in \mathbb{C}),
$$

which, in the special case when $\mu=n, n \in \mathbb{N}_{0}$, yields

$$
\left(\begin{array}{l}
\lambda \\
0
\end{array}\right):=1 \text { and }\left(\begin{array}{l}
\lambda \\
n
\end{array}\right):=\frac{\lambda(\lambda-1) \cdots(\lambda-n+1)}{n !}=\frac{(-1)^{n}(-\lambda)_{n}}{n !} \quad(n \in \mathbb{N}),
$$

where $(\lambda)_{\nu}(\lambda, \nu \in \mathbb{C})$ is the Pochhammer symbol defined, also in terms of the gamma function, by

$$
(\lambda)_{\nu}:=\frac{\Gamma(\lambda+\nu)}{\Gamma(\lambda)}= \begin{cases}1 & (\nu=0 ; \lambda \in \mathbb{C} \backslash\{0\}) \\ \lambda(\lambda+1) \cdots(\lambda+n-1) & (\nu=n \in \mathbb{N} ; \lambda \in \mathbb{C})\end{cases}
$$

it being understood conventionally that $(0)_{0}:=1$ and assumed that the $\Gamma$-quotient exists.

Let

$$
H_{n}=\sum_{r=1}^{n} \frac{1}{r}=\gamma+\psi(n+1)=\int_{0}^{1} \frac{1-t^{n}}{1-t} d t \quad\left(H_{0}:=0\right)
$$

be the $n t h$ harmonic number. Here, as usual, $\gamma$ denotes the Euler-Mascheroni constant and $\psi(z)$ is the Psi (or Digamma) function defined by

$$
\psi(z):=\frac{d}{d z}\{\log \Gamma(z)\}=\frac{\Gamma^{\prime}(z)}{\Gamma(z)} \quad \text { or } \quad \log \Gamma(z)=\int_{1}^{z} \psi(t) d t
$$

A generalized harmonic number $H_{n}^{(m)}$ of order $m$ is defined, for positive integers $n$ and $m$, as follows:

$$
H_{n}^{(m)}:=\sum_{r=1}^{n} \frac{1}{r^{m}}, \quad(m, n \in \mathbb{N}) \quad \text { and } \quad H_{0}^{(m)}:=0 \quad(m \in \mathbb{N})
$$

Tbilisi Mathematical Journal 8(2) (2015), pp. 195-209.

Tbilisi Centre for Mathematical Sciences.

Received by the editors: 18 May 2015. 
and

$$
\psi^{(n)}(z)=\frac{d^{n}}{d z^{n}}\{\psi(z)\}=\frac{d^{n+1}}{d z^{n+1}}\{\log \Gamma(z)\} \quad\left(n \in \mathbb{N}_{0}\right) .
$$

In the case of non integer values of the argument $z=\frac{r}{q}$, we may write the generalized harmonic numbers, $H_{z}^{(\alpha+1)}$, in terms of polygamma functions

$$
H_{\frac{r}{q}}^{(\alpha+1)}=\zeta(\alpha+1)+\frac{(-1)^{\alpha}}{\alpha !} \psi^{(\alpha)}\left(\frac{r}{q}+1\right), \frac{r}{q} \neq\{-1,-2,-3, \ldots\},
$$

where $\zeta(z)$ is the Riemann zeta function. When we encounter harmonic numbers at possible rational values of the argument of the form $H_{\frac{r}{q}}^{(\alpha)}$, they may be evaluated by an available relation in terms of the polygamma function $\psi^{(\alpha)}(z)$ or, for rational arguments $z=\frac{r}{q}$, and we also define

$$
H_{\frac{r}{q}}^{(1)}=\gamma+\psi\left(\frac{r}{q}+1\right), \text { and } H_{0}^{(\alpha)}=0
$$

The evaluation of the polygamma function $\psi^{(\alpha)}\left(\frac{r}{a}\right)$ at rational values of the argument can be explicitly done via a formula as given by Kölbig [8], or Choi and Cvijović [2] in terms of the polylogarithmic or other special functions. Some specific values are given as follows:

$$
\begin{gathered}
H_{-\frac{1}{2}}^{(1)}=-2 \ln 2, \quad H_{-\frac{1}{2}}^{(2)}=-2 \zeta(2), \\
H_{-\frac{3}{4}}^{(1)}=-\frac{\pi}{2}-3 \ln 2, \text { and } H_{-\frac{3}{4}}^{(2)}=-8 G-5 \zeta(2) .
\end{gathered}
$$

Many others are listed in the books[15], [22] and [23]. In this paper we will develop identities, closed form representations of alternating harmonic numbers and reciprocal binomial coefficients of the form

$$
S_{k}(p)=\sum_{n=1}^{\infty} \frac{(-1)^{n+1} H_{n}}{n^{p}\left(\begin{array}{c}
n+k \\
k
\end{array}\right)},
$$

for $p=0,1$ and 2 .

While there are many results for sums of harmonic numbers with positive terms, there are fewer results for sums of the type (1.1). Let us define the alternating zeta function

$$
\bar{\zeta}(z):=\sum_{n=1}^{\infty} \frac{(-1)^{n+1}}{n^{z}}=\left(1-2^{1-z}\right) \zeta(z)
$$

with $\bar{\zeta}(1)=\ln 2$, and

$$
S_{p, q}^{+-}=\sum_{n=1}^{\infty} \frac{(-1)^{n+1} H_{n}^{(p)}}{n^{q}} .
$$

Sitaramachandrarao [11] gave, for $1+q$ an odd integer,

$$
2 S_{1, q}^{+-}=(1+q) \bar{\zeta}(1+q)-\zeta(1+q)-2 \sum_{j=1}^{\frac{q}{2}-1} \bar{\zeta}(2 j) \zeta(1+q-2 j)
$$


and, in another special case, gave the integral

$$
S_{1,1+2 q}^{+-}=\int_{0}^{1} \frac{\ln ^{2 q}(x) \ln (1+x)}{x(1+x)} d x .
$$

In the case where $p$ and $q$ are both positive integers and $p+q$ is an odd integer, Flajolet and Salvy [7] gave the identity:

$$
\begin{aligned}
2 S_{p, q}^{+-}= & \left(1-(-1)^{p}\right) \zeta(p) \bar{\zeta}(q)+2 \sum_{i+2 k=q}\left(\begin{array}{c}
p+i-1 \\
p-1
\end{array}\right) \zeta(p+i) \bar{\zeta}(2 k) \\
& +\bar{\zeta}(p+q)-2 \sum_{j+2 k=p}\left(\begin{array}{c}
q+j-1 \\
q-1
\end{array}\right)(-1)^{j} \bar{\zeta}(q+j) \bar{\zeta}(2 k),
\end{aligned}
$$

where $\bar{\zeta}(0)=\frac{1}{2}, \bar{\zeta}(1)=\ln 2, \zeta(1)=0$, and $\zeta(0)=-\frac{1}{2}$ in accordance with the analytic continuation of the Riemann zeta function. Flajolet and Salvy [7], further gave some specific examples, such as

$$
\sum_{n=1}^{\infty} \frac{(-1)^{n+1} H_{n}}{2 n+1}=\frac{\pi \ln 2}{2}-G
$$

where

$$
G:=\sum_{n=0}^{\infty} \frac{(-1)^{n}}{(2 n+1)^{2}} \approx .91596 \text { is Catalan's constant. }
$$

Some other interesting cases are given by Coffey [5]

$$
\sum_{n=1}^{\infty} \frac{(-1)^{n+1} H_{n}^{(2)}}{(n+1)^{2}}=\frac{65}{16} \zeta(4)+\zeta(2) \ln ^{2} 2-\frac{1}{6} \ln ^{4}(2)-\frac{7}{2} \zeta(3) \ln 2-4 L_{i_{4}}\left(\frac{1}{2}\right)
$$

where $L_{i_{4}}(\cdot)$ is the polylogarithm function, Coffey [6] also gave the expression

$$
\sum_{n=1}^{\infty} \frac{(-1)^{n+1} \psi^{(p)}(n+a+1)}{n^{q}}=(-1)^{q} \int_{0}^{1} \int_{0}^{1} \frac{x^{a+1} \ln ^{q-1}(y) \ln ^{p}(x)}{(1+x y)(1-x)} d x d y
$$

where $\psi^{(p)}(\cdot)$ is the polygamma function. Some results for finite sums of alternating harmonic numbers may be seen in the works of [1], [3], [4], [9], [12], [13], [14], [16], [24], [25], [26] and references therein. For results on alternating quadratic harmonic number sums see [17]. Some results for sums of positive terms may be seen in the works [10], [18], [19], [20] and [21].

The following lemma will be useful in the development of the main theorems.

Lemma 1.1. Let $r$ be a positive integer. Then:

$$
\sum_{j=1}^{r} \frac{(-1)^{j}}{j}=H_{\left[\frac{r}{2}\right]}-H_{r}
$$


where $[x]$ is the integer part of $x$. We also have the known results. For $0<t \leq 1$

$$
\ln ^{2}(1+t)=2 \sum_{n=1}^{\infty} \frac{(-t)^{n+1} H_{n}}{n+1}
$$

and when $t=1$,

$$
\begin{aligned}
\ln ^{2} 2 & =2 \sum_{n=1}^{\infty} \frac{(-1)^{n+1} H_{n}}{n+1}=\zeta(2)-2 L_{i_{2}}\left(\frac{1}{2}\right) \\
& =2 \sum_{n=1}^{\infty} \frac{s(n, 2)}{n !}
\end{aligned}
$$

where $s(n, 2)$ are Stirling numbers of the first kind.

$$
t \ln (1+t)=\sum_{n=1}^{\infty} \frac{(-t)^{n+1}}{n}
$$

hence

$$
\ln 2=\sum_{n=1}^{\infty} \frac{(-1)^{n+1}}{n}=\sum_{n=1}^{\infty} \frac{1}{n 2^{n}}=\frac{1}{2} \sum_{n=1}^{\infty} \frac{H_{n}}{2^{n}} .
$$

Proof. To prove (1.2) we write,

$$
\begin{aligned}
\sum_{j=1}^{r} \frac{(-1)^{j}}{j} & =\sum_{j=1}^{r} \frac{1}{j}+\sum_{j=1}^{\left[\frac{r}{2}\right]} \frac{1}{j}-2 \sum_{j=1}^{\left[\frac{2 r+1}{2}\right]} \frac{1}{j} \\
& =H_{r}+H_{\left[\frac{r}{2}\right]}-2 H_{\left[\frac{2 r+1}{2}\right]}, \text { since } r \text { is a positive integer } \\
& =H_{\left[\frac{r}{2}\right]}-H_{r} .
\end{aligned}
$$

Lemma 1.2. Let $r$ be a positive integer. Then

$$
\begin{aligned}
S_{r}: & =\sum_{n=1}^{\infty} \frac{(-1)^{n+1} H_{n}}{n+r}=\frac{(-1)^{r+1}}{2} \ln ^{2} 2+(-1)^{r}\left(2 H_{r-1}-H_{\left[\frac{r-1}{2}\right]}\right) \ln 2 \\
& +(-1)^{r} \sum_{j=1}^{r-1} \frac{1}{j}\left(H_{\left[\frac{j}{2}\right]}-H_{j}\right), \text { for } r \geq 2,
\end{aligned}
$$

and

$$
S_{0}=\sum_{n=1}^{\infty} \frac{(-1)^{n+1} H_{n}}{n}=\frac{1}{2} \zeta(2)-\frac{1}{2} \ln ^{2} 2, S_{1}=\sum_{n=1}^{\infty} \frac{(-1)^{n+1} H_{n}}{n+1}=\frac{1}{2} \ln ^{2} 2
$$


Proof. By a change of counter

$$
\begin{aligned}
S_{r} & :=\sum_{n=1}^{\infty} \frac{(-1)^{n+1} H_{n}}{n+r}=\sum_{n=2}^{\infty} \frac{(-1)^{n} H_{n-1}}{n+r-1}=\sum_{n=2}^{\infty} \frac{(-1)^{n}}{n+r-1}\left(H_{n}-\frac{1}{n}\right) \\
& =-\sum_{n=1}^{\infty} \frac{(-1)^{n+1} H_{n}}{n+r-1}+\sum_{n=1}^{r-1} \frac{(-1)^{n+1}}{n(n+r-1)} \\
& =-S_{r-1}+\sum_{n=1}^{r-1} \frac{(-1)^{n+1}}{r-1}\left(\frac{1}{n}-\frac{1}{n+r-1}\right) .
\end{aligned}
$$

From Lemma 1.1 and using the known results

$$
\begin{aligned}
S_{r} & =-S_{r-1}+\frac{1}{r-1}\left(\ln 2-(-1)^{r} \sum_{n=1}^{\infty} \frac{(-1)^{n}}{n}-\sum_{n=1}^{r-1} \frac{(-1)^{n}}{n}\right) \\
& =-S_{r-1}+\frac{\ln 2}{r-1}\left(1+(-1)^{r}\right)+\frac{(-1)^{r}}{r-1}\left(H_{\left[\frac{r-1}{2}\right]}-H_{r-1}\right),
\end{aligned}
$$

which, for $r \geq 2$, yields the recurrence relation

$$
S_{r}+S_{r-1}=\frac{\ln 2}{r-1}\left(1+(-1)^{r}\right)+\frac{(-1)^{r}}{r-1}\left(H_{\left[\frac{r-1}{2}\right]}-H_{r-1}\right) .
$$

By the subsequent reduction of the $S_{r}, S_{r-1}, S_{r-2}, \ldots S_{1}$ terms in (1.6), we arrive at the identity $(1.5)$.

It is of some interest to note that $S_{r}$ may be expanded in a slightly different way so that it gives rise to another unexpected harmonic series identity. This is pursued in the next lemma.

Lemma 1.3. For $r \in \mathbb{N}_{0}$, we have the identity

$$
\begin{aligned}
& V_{r} \quad: \quad= \sum_{j=1}^{\infty} \frac{H_{j+\frac{r-2}{2}}-H_{j+\frac{r-3}{2}}}{2(2 j-1)}=\frac{(-1)^{r+1}}{2} \ln ^{2} 2+(-1)^{r}\left(2 H_{r-1}-H_{\left[\frac{r-1}{2}\right]}\right) \ln 2 \\
&+ \frac{H_{\frac{r-1}{2}}}{2(r-1)}+(-1)^{r} H_{r-1}\left(H_{\left[\frac{r}{2}\right]}-H_{r}\right)-\frac{1}{8}\left(H_{\frac{r-2}{2}}^{2}-H_{\frac{r-3}{2}}^{2}+H_{\frac{r-2}{2}}^{(2)}-H_{\frac{r-3}{2}}^{(2)}\right) \\
&+(-1)^{r} \sum_{j=1}^{r-1} \frac{(-1)^{j+1}}{j}\left(H_{\left[\frac{r-j}{2}\right]}-H_{r-j}+\frac{j H_{j}}{j+1}\right) .
\end{aligned}
$$

Proof. By expansion

$$
S_{r}:=\sum_{n=1}^{\infty} \frac{(-1)^{n+1} H_{n}}{n+r}=\sum_{n=1}^{\infty}\left(\frac{H_{2 n}}{(2 n+r)(2 n+r-1)}-\frac{1}{2 n(2 n+r-1)}\right),
$$


since we know that $H_{2 n}=\frac{1}{2} H_{n}+\sum_{j=1}^{n} \frac{1}{2 j-1}$ then

$$
\begin{aligned}
S_{r}= & \frac{1}{2} \sum_{n=1}^{\infty} \frac{H_{n}}{(2 n+r)(2 n+r-1)}+\sum_{n=1}^{\infty} \sum_{j=1}^{n} \frac{1}{(2 j-1)(2 n+r)(2 n+r-1)} \\
& -\sum_{n=1}^{\infty} \frac{1}{2 n(2 n+r-1)} \\
= & \frac{1}{8}\left(H_{\frac{r-2}{2}}^{2}-H_{\frac{r-3}{2}}^{2}+H_{\frac{r-2}{2}}^{(2)}-H_{\frac{r-3}{2}}^{(2)}\right)-\frac{H_{\frac{r-1}{2}}}{2(r-1)} \\
& +\sum_{n=1}^{\infty} \sum_{j=1}^{n} \frac{1}{(2 j-1)(2 n+r)(2 n+r-1)} .
\end{aligned}
$$

For an arbitrary double sequence $Y_{k, l}$ we have that

$$
\sum_{k=0}^{\infty} \sum_{l=0}^{k} Y_{k, l}=\sum_{l=0}^{\infty} \sum_{k=0}^{\infty} Y_{k, l+k}
$$

and from (1.9)

$$
\begin{aligned}
V_{r} & =\sum_{n=1}^{\infty} \sum_{j=1}^{n} \frac{1}{(2 j-1)(2 n+r)(2 n+r-1)} \\
& =\sum_{j=1}^{\infty} \sum_{n=0}^{\infty} \frac{1}{(2 j-1)(2 n+2 j+r)(2 n+2 j+r-1)} \\
& =\sum_{j=1}^{\infty} \frac{1}{2(2 j-1)}\left(H_{j+\frac{r-2}{2}}-H_{j+\frac{r-3}{2}}\right) .
\end{aligned}
$$

Upon utilizing (1.9) and the known result (1.5) for $S_{r}$, we are able to write

$$
\begin{aligned}
\sum_{j=1}^{\infty} \frac{1}{2(2 j-1)}\left(H_{j+\frac{r-2}{2}}-H_{j+\frac{r-3}{2}}\right)= & S_{r}+\frac{H_{\frac{r-1}{2}}}{2(r-1)} \\
& -\frac{1}{8}\left(H_{\frac{r-2}{2}}^{2}-H_{\frac{r-3}{2}}^{2}+H_{\frac{r-2}{2}}^{(2)}-H_{\frac{r-3}{2}}^{(2)}\right) .
\end{aligned}
$$

Substituting for $S_{r}$ and upon simplification we have the result (1.7) for $V_{r}$.

Remark 1.4. We note that Lemma 1.3 states the difference of two diverging harmonic series 
produce a converging series:

$$
\begin{aligned}
& V_{0}=\sum_{j=1}^{\infty} \frac{H_{j}-H_{j-\frac{1}{2}}}{2 j+1}=\zeta(2)-2 \ln 2, \\
& V_{1}=\sum_{j=1}^{\infty} \frac{H_{j+\frac{1}{2}}-H_{j}}{2 j+1}=\frac{1}{2} \zeta(2)+2 \ln 2-2, \\
& V_{2}=\sum_{j=1}^{\infty} \frac{H_{j+1}-H_{j+\frac{1}{2}}}{2 j+1}=1-\frac{1}{2} \zeta(2), \text { and } \\
& V_{3}=\sum_{j=1}^{\infty} \frac{H_{j+\frac{3}{2}}-H_{j+1}}{2 j+1}=\frac{1}{2} \zeta(2)-\frac{2}{3}
\end{aligned}
$$

The next several theorems relate the main results of this investigation, namely the closed form and integral representation of (1.1).

\section{Closed form and Integral identities}

We now prove the following theorems.

Theorem 2.1. Let $k$ be real positive integer. Then from (1.1) with $p=0$ we have

$$
\begin{gathered}
S_{k}(0)=\sum_{n=1}^{\infty} \frac{(-1)^{n+1} H_{n}}{\left(\begin{array}{c}
n+k \\
k
\end{array}\right)}=2^{k-2} k \ln ^{2} 2 \\
-\sum_{r=1}^{k} r\left(\begin{array}{c}
k \\
r
\end{array}\right)\left(\begin{array}{c}
\left(2 H_{r-1}-H_{\left[\frac{r-1}{2}\right]}\right) \ln 2+H_{r-1}\left(H_{\left[\frac{r}{2}\right]}-H_{r}\right) \\
-\sum_{j=1}^{r-1} \frac{(-1)^{j}}{j}\left(H_{\left[\frac{r-j}{2}\right]}-H_{r-j}+\frac{j H_{j}}{j+1}\right)
\end{array}\right) .
\end{gathered}
$$

Proof. Consider the expansion

$$
\begin{aligned}
& S_{k}(0)=\sum_{n=1}^{\infty} \frac{(-1)^{n+1} H_{n}}{\left(\begin{array}{c}
n+k \\
k
\end{array}\right)}=\sum_{n=1}^{\infty} \frac{(-1)^{n+1} k ! H_{n}}{(n+1)_{k}} \\
= & \sum_{n=1}^{\infty}(-1)^{n+1} k ! H_{n} \sum_{r=1}^{k} \frac{\Lambda_{r}}{n+r},
\end{aligned}
$$

where

$$
\Lambda_{r}=\lim _{n \rightarrow-r}\left\{\frac{n+r}{\prod_{r=1}^{k} n+r}\right\}=\frac{(-1)^{r+1} r}{k !}\left(\begin{array}{l}
k \\
r
\end{array}\right) .
$$


Hence

$$
\begin{aligned}
S_{k}(0) & =\sum_{r=1}^{k}(-1)^{r+1} r\left(\begin{array}{l}
k \\
r
\end{array}\right) \sum_{n=1}^{\infty} \frac{(-1)^{n+1} H_{n}}{n+r} \\
& =\sum_{r=1}^{k}(-1)^{r+1} r\left(\begin{array}{l}
k \\
r
\end{array}\right) S_{r} .
\end{aligned}
$$

From Lemma 1.2, (1.5) substituting into (2.2),

$$
\begin{aligned}
S_{k}(0)= & \sum_{r=1}^{k}(-1)^{r+1} r\left(\begin{array}{l}
k \\
r
\end{array}\right)\left(\begin{array}{c}
\frac{(-1)^{r+1}}{2} \ln ^{2} 2+(-1)^{r}\left(2 H_{r-1}-H_{\left[\frac{r-1}{2}\right]}\right) \ln 2 \\
+(-1)^{r} H_{r-1}\left(H_{\left[\frac{r}{2}\right]}-H_{r}\right) \\
-(-1)^{r} \sum_{j=1}^{r-1} \frac{(-1)^{j}}{j}\left(H_{\left[\frac{r-j}{2}\right]}-H_{r-j}+\frac{j H_{j}}{j+1}\right)
\end{array}\right) \\
= & -2_{r=1}^{k-2} k \ln ^{2} 2 \\
& \left.-\sum^{k}\right)\left(\begin{array}{c}
\left(2 H_{r-1}-H_{\left[\frac{r-1}{2}\right]}\right) \ln 2+H_{r-1}\left(H_{\left[\frac{r}{2}\right]}-H_{r}\right) \\
-\sum_{j=1}^{r-1} \frac{(-1)^{j}}{j}\left(H_{\left[\frac{r-j}{2}\right]}-H_{r-j}+\frac{j H_{j}}{j+1}\right)
\end{array}\right),
\end{aligned}
$$

and Theorem 2.1 follows.

The other two cases of $S_{k}(1), S_{k}(2)$ can be evaluated in a similar fashion. We list the results in the next corollary.

Corollary 2.2. Under the assumptions of Theorem 2.1, we have,

$$
\begin{gathered}
S_{k}(1)=\sum_{n=1}^{\infty} \frac{(-1)^{n+1} H_{n}}{n\left(\begin{array}{c}
n+k \\
k
\end{array}\right)}=\frac{1}{2} \zeta(2)+\left(2^{k-1}-1\right) \ln ^{2} 2 \\
+\sum_{r=1}^{k} r\left(\begin{array}{c}
k \\
r
\end{array}\right)\left(\begin{array}{c}
\left(2 H_{r-1}-H_{\left[\frac{r-1}{2}\right]}\right) \ln 2+H_{r-1}\left(H_{\left[\frac{r}{2}\right]}-H_{r}\right) \\
-\sum_{j=1}^{r-1} \frac{(-1)^{j}}{j}\left(H_{\left[\frac{r-j}{2}\right]}-H_{r-j}+\frac{j H_{j}}{j+1}\right)
\end{array}\right),
\end{gathered}
$$

and

$$
S_{k}(2)=\sum_{n=1}^{\infty} \frac{(-1)^{n+1} H_{n}}{n^{2}\left(\begin{array}{c}
n+k \\
k
\end{array}\right)}=\frac{5}{8} \zeta(3)-\frac{1}{2}\left(\zeta(2)-\ln ^{2} 2\right) H_{k}
$$




$$
+\sum_{r=1}^{k} \frac{1}{r}\left(\begin{array}{c}
k \\
r
\end{array}\right)\left(\begin{array}{c}
\frac{1}{2} \ln ^{2} 2-\left(2 H_{r-1}-H_{\left[\frac{r-1}{2}\right]}\right) \ln 2-H_{r-1}\left(H_{\left[\frac{r}{2}\right]}-H_{r}\right) \\
+\sum_{j=1}^{r-1} \frac{(-1)^{j}}{j}\left(H_{\left[\frac{r-j}{2}\right]}-H_{r-j}+\frac{j H_{j}}{j+1}\right)
\end{array}\right) .
$$

Proof. The proof follows directly from Theorem 2.1 and using the same technique.

It is possible to represent the alternating harmonic number sums (2.1), (2.3) and (2.4) in terms of an integral, this is developed in the next theorem.

Theorem 2.3. Let $k$ be a positive integer. Then we have:

$$
\begin{aligned}
& S_{k}(0)=\sum_{n=1}^{\infty} \frac{(-1)^{n+1} H_{n}}{\left(\begin{array}{c}
n+k \\
k
\end{array}\right)}=-k \int_{0}^{1} \int_{0}^{1} \frac{x y(1-x)^{k-1}}{(1-y)(1+x y)} d x d y \\
& =-k \int_{0}^{1} \int_{0}^{1} \frac{x(1-x)^{k-1} \ln (1-y)}{(1+x y)^{2}} d x d y \\
& =\left(\frac{1-k}{2}\right) \ln (2)-\frac{k}{1+k} \int_{0}^{1} \frac{(k+y){ }_{2} F_{1}\left[\begin{array}{c}
1,1 \\
2+k
\end{array} \mid-y\right] \ln (1+y)}{(1+y)^{2}} d y .
\end{aligned}
$$

Proof. Let $j \in \mathbb{N}_{0}, k \in \mathbb{N}$ and $|t| \leq 1$. Consider the expansion

$$
\begin{aligned}
\sum_{n=1}^{\infty} \frac{t^{n}}{\left(\begin{array}{c}
n+k \\
k
\end{array}\right)\left(\begin{array}{c}
n+j \\
j
\end{array}\right)} & =k j \sum_{n=1}^{\infty} \frac{t^{n} \Gamma(k) \Gamma(n+1) \Gamma(j) \Gamma(n+1)}{\Gamma(n+k+1) \Gamma(n+j+1)} \\
& =k j \sum_{n=1}^{\infty} t^{n} B(k, n+1) B(j, n+1),
\end{aligned}
$$

where $B(\cdot, \cdot)$ is the classical Beta function. Therefore

$$
\begin{aligned}
\sum_{n=1}^{\infty} \frac{t^{n}}{\left(\begin{array}{c}
n+k \\
k
\end{array}\right)\left(\begin{array}{c}
n+j \\
j
\end{array}\right)} & =k j \int_{0}^{1} \int_{0}^{1}(1-x)^{k-1}(1-y)^{j-1} \sum_{n=1}^{\infty}(t x y)^{n} d x d y . \\
& =k j \int_{0}^{1} \int_{0}^{1} \frac{(1-x)^{k-1}(1-y)^{j-1} t x y}{1-t x y} d x d y .
\end{aligned}
$$


The next step is to differentiate both sides with respect to $j$ then put $j=0$ and $t=-1$, from which we obtain

$$
S_{k}(0)=\sum_{n=1}^{\infty} \frac{(-1)^{n+1} H_{n}}{\left(\begin{array}{c}
n+k \\
k
\end{array}\right)}=-k \int_{0}^{1} \int_{0}^{1} \frac{x y(1-x)^{k-1}}{(1-y)(1+x y)} d x d y:=J_{k}(0) .
$$

Hence (2.5) follows. The identity (2.6) follows from the expansion

$$
\begin{aligned}
\sum_{n=1}^{\infty} \frac{t^{n}}{\left(\begin{array}{c}
n+k \\
k
\end{array}\right)\left(\begin{array}{c}
n+j \\
j
\end{array}\right)} & =k \int_{0}^{1} \int_{0}^{1} \frac{(1-x)^{k-1}(1-y)^{j}}{y} \sum_{n=1}^{\infty} n(t x y)^{n} d x d y \\
& =k \int_{0}^{1} \int_{0}^{1} \frac{(1-x)^{k-1}(1-y)^{j} t x}{(1-t x y)^{2}} d x d y
\end{aligned}
$$

differentiating and making the appropriate substitutions (2.6) follows. Moreover from (2.6)

$$
\begin{aligned}
S_{k}(0)= & -k \int_{0}^{1} \int_{0}^{1} \frac{x(1-x)^{k-1} \ln (1-y)}{(1+x y)^{2}} d x d y=(k-1) \int_{0}^{1} \frac{\ln (1-y)}{(1+y)^{2}} d y \\
& -\frac{k}{1+k} \int_{0}^{1} \frac{(k+y){ }_{2} F_{1}\left[\begin{array}{c}
1,1 \\
2+k
\end{array} \mid-y\right] \ln (1+y)}{(1+y)^{2}} d y
\end{aligned}
$$

which is the identity $(2.7)$. If we let

$$
I_{k}(0):=\frac{k}{1+k} \int_{0}^{1} \frac{(k+y){ }_{2} F_{1}\left[\begin{array}{c}
1,1 \\
2+k
\end{array} \mid-y\right] \ln (1+y)}{(1+y)^{2}} d y
$$

we note that $I_{k}(0)$ may be written explicitly in terms of the right hand side of $(2.1)$, moreover we note that

$$
I_{k}(0)+J_{k}(0)=\left(\frac{1-k}{2}\right) \ln 2 .
$$

Similar integral representations can be evaluated for $S_{k}(1)$ and $S_{k}(2)$, the results are recorded in the next theorem.

Theorem 2.4. Let the conditions of Theorem 2.3 hold. Then we have:

$$
S_{k}(1)=\sum_{n=1}^{\infty} \frac{(-1)^{n+1} H_{n}}{n\left(\begin{array}{c}
n+k \\
k
\end{array}\right)}=-\int_{0}^{1} \int_{0}^{1} \frac{(1-x)^{k} \ln (1-y)}{1+x y} d x d y,
$$




$$
\begin{gathered}
=\int_{0}^{1} \frac{(1-x)^{k} \ln (1+x)}{x(1+x)} d x \\
=\frac{1}{1+k}{ }_{3} F_{2}\left[\begin{array}{c|c}
1,1,1 & -1 \\
2,2+k & -1
\end{array}\right. \\
+2^{k-1}\left(2 \ln 2 H_{k}-\ln ^{2} 2-H_{k}^{2}-H_{k}^{(2)}+k_{4} F_{3}\left[\begin{array}{c|c}
1,1,1,1-k & 1 \\
2,2,2 & \frac{1}{2}
\end{array}\right]\right) .
\end{gathered}
$$

Also

$$
S_{k}(1)=-k \int_{0}^{1} \int_{0}^{1} \frac{x(1-x)^{k} \ln (1-y)}{1+x y} d x d y
$$

For $S_{k}(2)$,

$$
S_{k}(2)=\sum_{n=1}^{\infty} \frac{(-1)^{n+1} H_{n}}{n^{2}\left(\begin{array}{c}
n+k \\
k
\end{array}\right)}=-k \int_{0}^{1} \int_{0}^{1} \frac{(1-x)^{k-1} \ln (1-y) \ln (1+x y)}{y} d x d y,
$$

and also,

$$
\begin{aligned}
S_{k}(2) & =-\int_{0}^{1} \int_{0}^{1} \frac{(1-x)^{k} \ln (1-y)}{1+x y} d x d y \\
& =-\frac{1}{1+k} \int_{0}^{1}{ }_{2} F_{1}\left[\begin{array}{c}
1,1 \\
2+k
\end{array} \mid-y\right] \ln (1+y) d y .
\end{aligned}
$$

Proof. The proof follows from the same pattern as that employed in Theorem 2.3.

Example 2.5. Some illustrative examples are given as follows:

$$
\begin{aligned}
S_{4}(0) & =\sum_{n=1}^{\infty} \frac{(-1)^{n+1} H_{n}}{\left(\begin{array}{c}
n+4 \\
4
\end{array}\right)}=16 \ln ^{2} 2-\frac{176}{3} \ln 2+\frac{298}{9} \\
& =-\frac{3}{2} \ln (2)-\frac{4}{5} \int_{0}^{1} \frac{(4+y){ }_{2} F_{1}\left[\begin{array}{c}
1,1 \\
6
\end{array} \mid-y\right] \ln (1+y)}{(1+y)^{2}} d y \\
S_{4}(1) & =\sum_{n=1}^{\infty} \frac{(-1)^{n+1} H_{n}}{n\left(\begin{array}{c}
n+4 \\
4
\end{array}\right)}=\frac{1}{2} \zeta(2)-8 \ln ^{2} 2+\frac{68}{3} \ln 2-\frac{451}{36} \\
& =\int_{0}^{1} \frac{(1-x)^{4} \ln (1+x)}{x(1+x)} d x, \text { and }
\end{aligned}
$$




$$
\begin{aligned}
S_{4}(2) & =\sum_{n=1}^{\infty} \frac{(-1)^{n+1} H_{n}}{n^{2}\left(\begin{array}{c}
n+4 \\
4
\end{array}\right)}=\frac{5}{8} \zeta(3)-\frac{25}{24} \zeta(2)+\frac{16}{3} \ln ^{2} 2-\frac{28}{3} \ln 2+\frac{727}{144} \\
& =-\frac{1}{5} \int_{0}^{1}{ }_{2} F_{1}\left[\begin{array}{c|c}
1,1 & -y \\
6 & -y
\end{array}\right] \ln (1+y) d y .
\end{aligned}
$$

Generally speaking it should be possible to obtain explicit identities of

$$
\sum_{n=1}^{\infty} \frac{(-t)^{n+1} H_{n}}{n^{p}\left(\begin{array}{c}
n+4 \\
4
\end{array}\right)}, 0<t \leq 1
$$

For $t=\frac{1}{2}$,

$$
\sum_{n=1}^{\infty} \frac{\left(-\frac{1}{2}\right)^{n+1} H_{n}}{\left(\begin{array}{c}
n+4 \\
4
\end{array}\right)}=54\left(\ln ^{2} 3+\ln ^{2} 2\right)-108 \ln 2 \ln 3-198 \ln \frac{3}{2}+\frac{643}{9} .
$$

\section{Some Observations and Concluding remarks}

The alternating sums of harmonic numbers $S_{k}(p)$, for $p=0,1$ and 2 have been successfully represented in integral form and in terms of zeta functions, harmonic numbers and ln functions. It may also be possible to represent the sums

$$
S_{k}(p, q, r)=\sum_{n=1}^{\infty} \frac{(-1)^{n+1} H_{n}^{(r)}}{n^{p}\left(\begin{array}{c}
n+k \\
k
\end{array}\right)^{q}}
$$

in closed form, this work is currently under investigation. It does appear however, that there is an impasse with the representation $S_{k}(3)$ in closed form. This is related to the fact that

$$
V(0):=\sum_{n=1}^{\infty} \frac{(-1)^{n+1} H_{n}}{n^{3}}
$$

has, currently no closed form representation. In one scenario, $S_{k}(3)$ necessitates the evaluation of the difference of two diverging sums

$$
\Theta(\alpha)=\sum_{n=1}^{\infty} \frac{H_{n}^{(\alpha)}-H_{n-\frac{1}{2}}^{(\alpha)}}{2 n+1}
$$

for $\alpha=3$. Currently we have the known identities

$$
\begin{aligned}
& \Theta(1)=\zeta(2)-2 \ln 2, \Theta(2)=(3 \ln 2-2) \zeta(2) \\
& \Theta(3)=17 \zeta(4)-(6+7 \ln 2) \zeta(3)+4 \ln ^{2} 2 \zeta(2)-\frac{2}{3} \ln ^{4} 2-16 L_{i_{4}}\left(\frac{1}{2}\right) \\
& \Theta(4)=2 \zeta(2) \zeta(3)-(15 \ln 2-14) \zeta(4)
\end{aligned}
$$


and $\Theta(2 m)$ for $m \in \mathbb{N}$. Similarly

$$
\begin{aligned}
\sum_{n=1}^{\infty} \frac{(-1)^{n+1} H_{n}}{n^{3}}= & \frac{1}{8}(5 \zeta(4)+(6-7 \ln 2) \zeta(3)+\Theta(3)) \\
= & \frac{11}{4} \zeta(4)-\frac{7}{4} \ln 2 \zeta(3)+\frac{1}{2} \ln ^{2} 2 \zeta(2) \\
& -\frac{1}{12} \ln ^{4} 2-2 L_{i_{4}}\left(\frac{1}{2}\right) .
\end{aligned}
$$

While $V(0)$ appears to have no "closed" form representation, quite remarkably two subsequent terms $V(r-1)$ and $V(r)$ can be represented in closed form in the following way.

Lemma 3.1. The following representation follows,

$$
\begin{gathered}
V(r-1)+V(r):=\sum_{n=1}^{\infty}(-1)^{n+1} H_{n}\left(\frac{1}{(n+r-1)^{3}}+\frac{1}{(n+r)^{3}}\right) \\
=\frac{\ln 2}{(r-1)^{3}}+\sum_{j=1}^{3} \frac{(-1)^{j}}{2^{j}(r-1)^{4-j}}\left(H_{\frac{r-1}{2}}^{(j)}-H_{\frac{r-2}{2}}^{(j)}\right), \text { for } r \geq 2,
\end{gathered}
$$

and for $r=1$

$$
V(0)+V(1):=\sum_{n=1}^{\infty}(-1)^{n+1} H_{n}\left(\frac{1}{n^{3}}+\frac{1}{(n+1)^{3}}\right)=\frac{7}{8} \zeta(4)
$$

Proof. Let

$$
\begin{aligned}
V(r-1)+V(r) & =\sum_{n=1}^{\infty}(-1)^{n+1} H_{n}\left(\frac{1}{(n+r-1)^{3}}+\frac{1}{(n+r)^{3}}\right) \\
& =\sum_{n=0}^{\infty}(-1)^{n+1} \frac{\left(H_{n}-H_{n+1}\right)}{(n+r)^{3}}=\sum_{n=0}^{\infty} \frac{(-1)^{n}}{(n+1)(n+r)^{3}} \\
= & \sum_{n=0}^{\infty} \frac{(-1)^{n}}{(r-1)^{3}}\left(\frac{1}{n+1}-\frac{1}{n+r}-\frac{r-1}{(n+r)^{2}}-\frac{(r-1)^{2}}{(n+r)^{3}}\right)
\end{aligned}
$$

and the identity of Lemma 3.1 follows.

\section{References}

[1] J. M. Borwein, I. J. Zucker and J. Boersma. The evaluation of character Euler double sums. Ramanujan J. 15 (2008), 377-405. 
[2] J. Choi and D. Cvijović. Values of the polygamma functions at rational arguments. J. Phys. A: Math. Theor. 40 (2007), 15019-15028; Corrigendum, ibidem, 43 (2010), 239801 (1 p).

[3] J. Choi. Finite summation formulas involving binomial coefficients, harmonic numbers and generalized harmonic numbers. J. Inequal. Appl. 49 (2013), 11p.

[4] J. Choi, and H. M. Srivastava. Some summation formulas involving harmonic numbers and generalized harmonic numbers. Math. Comput. Modelling. 54 (2011), 2220-2234.

[5] M. W. Coffey. On one dimensional digamma and polygamma series related to the evaluation of Feynman diagrams. J. Comput. Appl. Math. 183 (2005), 84-100.

[6] M. W. Coffey and N. Lubbers. On generalized harmonic number sums. Appl. Math. Comput. 217 (2010), 689-698.

[7] P. Flajolet and B. Salvy. Euler sums and contour integral representations. Exp. Math. 7 (1998), 15-35.

[8] K. Kölbig. The polygamma function $\psi(x)$ for $x=1 / 4$ and $x=3 / 4$. J. Comput. Appl. Math. 75 (1996), 43-46.

[9] H. Liu and W. Wang. Harmonic number identities via hypergeometric series and Bell polynomials. Integral Transforms Spec. Funct. 23 (2012), 49-68.

[10] T. Rassias and H. M. Srivastava. Some classes of infinite series associated with the Riemann zeta and polygamma functions and generalized harmonic numbers. Appl. Math. Comput. 131 (2002), 593-605.

[11] R. Sitaramachandrarao. A formula of S. Ramanujan. J. Number Theory 25 (1987), 1-19.

[12] A. Sofo. Sums of derivatives of binomial coefficients. Adv. in Appl. Math. 42 (2009), 123-134.

[13] A. Sofo. Integral forms associated with harmonic numbers. Appl. Math. Comput. 207 (2009), 365-372.

[14] A. Sofo. Integral identities for sums. Math. Commun. 13 (2008), 303-309.

[15] A. Sofo. Computational Techniques for the Summation of Series. Kluwer Academic/Plenum Publishers, New York, 2003.

[16] A. Sofo and H. M. Srivastava. Identities for the harmonic numbers and binomial coefficients, Ramanujan J. 25 (2011), 93-113.

[17] A. Sofo. Quadratic alternating harmonic number sums. J. Number Theory. 154 (2015), 144159.

[18] A. Sofo. Harmonic numbers and double binomial coefficients. Integral Transforms Spec. Funct. 20 (2009), 847-857.

[19] A. Sofo. Harmonic sums and intergal representations. J. Appl. Anal. 16 (2010), 265-277. 
[20] A. Sofo. Summation formula involving harmonic numbers. Analysis Math. 37 (2011), 51-64.

[21] A. Sofo and H. M. Srivastava. A family of shifted harmonic sums. Ramanujan J. 37 (2015), 89-108.

[22] H. M. Srivastava and J. Choi,.Series Associated with the Zeta and Related Functions. Kluwer Academic Publishers, London, 2001.

[23] H. M. Srivastava and J. Choi. Zeta and q-Zeta Functions and Associated Series and Integrals, Elsevier Science Publishers, Amsterdam, London and New York, 2012.

[24] W. Wang and C. Jia. Harmonic number identities via the Newton-Andrews method. Ramanujan J. 35 (2014), 263-285.

[25] C. Wei, D. Gong and Q. Wang. Chu-Vandermonde convolution and harmonic number identities. Integral Transforms Spec. Funct. 24 (2013), 324-330.

[26] D. Y. Zheng. Further summation formulae related to generalized harmonic numbers. J. Math. Anal. Appl. 335(1) (2007), 692-706. 